\title{
Global Flip \& Other Studies Workgroup Report
}

\author{
Eric Archambault, Colleen Campbell, Lorcan Dempsey, Roy Kaufman, Kamran Naim, Ralf Schimmer, \\ Wim van der Stelt, Caroline Sutton, Megan Wacha
}

\begin{abstract}
In their report, delegates of the Who Decides? workgroup of the OSI2016 conference put forth three proposals in which key stakeholders might convene to enact an economically viable and sustainable transformation of the current scholarly communications system to one of open access. The "Global Flip" workgroup of OSI2017 discussed the previous year's "Proposal 3: Transformation: a "global flip" of research journals to open access" in which "libraries, publishers, and funders, convened by an organization with global standing, come together to redirect subscription funding toward transforming existing journals to open access publication." Tasked with creating broad action plans for further research into the feasibility and impact of such a transformation, we identify a number of driving forces in the envisioned transformation, which could be further developed to assure its ultimate success as well as possible barriers to its desired fruition and suggested actions to remove them.
\end{abstract}

\section{OSI2017 Workgroup Question}

Following up on the research ideas proposed by OSI2016 delegates, this workgroup will create broad action plans for a variety of studies, beginning with the global flip, moving next to embargos, and also including publisher services disaggregation and an assessment of open impacts, if possible-how fast, how even, systemic pressures, and so on (referencing the OSI2016 workgroup papers on these various topics). Detailed study protocols aren't expected, but rather an outline of what to prioritize and how to conduct this work without necessarily relying on large grants from neutral parties. With regard to the global flip, this research is needed to help answer the question of whether a flip using article processing charges (APCs) is the right model to pursue (given concerns, for instance, about how this might affect access in the global south).

\section{Focus of the OSI 2017}

\section{Workgroup}

The question(s) posed for this workgroup were broad and potentially too diverse to be covered during the course of the conference. For this reason, members of the workgroup decided to focus on the global flip. While recognizing that there is some opposition to the concept of a global flip, members of the group further agreed to assume the possibility of a global flip, for the sake of discussion.

\section{Global Flip Defined}

In its report, the OSI 2016 Who Decides? workgroup describes the necessity for all stakeholders in the current scholarly

(C) 2017 OSI2017 Global Flip and Other Studies Workgroup. This open access article is distributed under the Creative Commons Attribution 4.0 International License. This document reflects the combined input of the authors listed here (in alphabetical order by last name) as well as contributions from other OSI2017 delegates. The findings and recommendations expressed herein do not necessarily reflect the opinions of the individual authors listed here, nor their agencies, trustees, officers, or staff. 
communications ecosystem-funding agencies, libraries, universities, publishers, and researchers - to come together in concerted efforts in order to achieve the overarching goals of enabling "an economically feasible, sustainable move to open access (OA), while preserving the quality and ensuring preservation and access" and to "improve the creation and dissemination of new knowledge". The report outlines three proposals that offer the opportunity of immediate concerted action and transformative results:

1. Evaluation: re-assessing criteria for academic tenure and promotion 2. Incubation: nurturing alternative, community-driven publishing models 3. Transformation: facilitating a "global flip" of research journals from subscription-based to OA.

The OSI2016 report further describes "Proposal 3: Transformation: a "global flip" of research journals to open access":

In this model, libraries, publishers, and funders, convened by an organization with global standing, come together to redirect subscription funding toward transforming existing journals to open access publication. The idea is to serve the public good and the commons of information, by reusing the same funds that today are spent to provide access to a limited audience. Among the current examples are:

- SCOAP3, which has established a global funding consortium of libraries and research funders under the auspices of CERN to convert a significant portion of the literature of high-energy physics to open access, at no burden to authors, at a cost-perarticle considerably lower than existing open access "APC" arrangements.

- A number of publishers and national-level library consortia in Europe are developing so-called offsetting pilot agreements in which, as part of the contracts to purchase access from providers to a portfolio of journals and without significant additional cost, an institution's article output is published as open access.

- The OA2020 initiative, recently launched by the Max Planck Society, is soliciting formal "expressions of interest" whereby libraries (on an individual or, preferably, national level) can publicly declare their intention to migrate from subscriptions to open access. A likely mechanism for this is the offsetting model described above, although other methods could be explored, including combining offsetting with the cultivation of alternative models.

- LIBRARIA is a collective of anthropology, archaeology, and social studies of science journals and learned societies that have teamed with the Public Knowledge Project and the SPARC to develop cooperative alternatives that go beyond APC-funded open access. LIBRARIA aims to develop an economically viable approach that brings together libraries, journals, and learned societies to cooperate, seeking more efficient allocation of resources, 
while advancing open access

and the quality of scholarship.

("Report from the Who Decides?

Workgroup";

http:/ /dx.doi.org/10.13021/G8P30V)

The Global Flip workgroup at OSI 2017 brought together the perspective of various stakeholders and observers and rooted its considerations in the understanding that the proposed "flip" may be simply defined as a large-scale conversion of a critical mass of the current corpus of scholarly journals from a subscription economy to business models that would ensure immediate open access to the published research outputs.

The global flip is therefore not, in and of itself, an open access business model; rather it is a means to open the path toward any number of OA publishing models and is viewed as a complement to existing and new open access initiatives.

Such a transformation is seen as immediately actionable in that, rather than requiring new infrastructure or investment, it makes use of the economic resources (i.e. library budgets) and scholarly communications infrastructure (publisher services) already in place, grounding the shift in terms of budgetary policy (repurposing existing funds).

In an effort to better evaluate what further research might be required to test the feasibility and impact of a large-scale conversion of today's scholarly journals to open access, the workgroup discussed and present here the driving forces of the envisioned transformation that might be further developed to assure its ultimate success, as well as possible barriers to its desired fruition and suggested actions to remove them.

\section{Drivers of the Global Flip}

Momentum around the large-scale transformation of the existing corpus of scholarly journals from subscription-based ("paywall") to open access ("OA") is being driven by a number of factors:

- Public Good: Immediate access to knowledge is an overarching goal that serves the interest of society at large and aligns with an increasing number of policies of philanthropic and governmental funding agencies.

- Speed of Implementation: Since launch of the Budapest Open Access Initiative in 2002, new, alternative open access publishing models (OSI2016 Proposal 2) have brought laudable but slow results with only around $15 \%$ of scholarly outputs available open access immediately on publication today; at this rate it will be decades before the goal of universal open access to the world's research will be achieved. Using current scholarly communications practices and infrastructure as leverage, i.e. article output and journal structure, the "global flip" represents an agile and rapidly adoptable pathway to open access requiring no substantial new investment from the community.

- Sensitivity to current scholar behavior: While efforts in re-assessing criteria for academic tenure and promotion may be pursued (OSI2016 Proposal 1), scholars currently rely on the structure and services provided by publishers of "traditional" journals. Additionally, selfarchiving mandates and practices ("Green" OA) are inconsistent and, as yet, ineffective for rendering versions of scholarly outputs accessible open access at scale. Rather than 
forcing changes in researcher behavior and practices, the "global flip" scenario leaves the researcher to continue publishing and disseminating their research according to their current practices and in the journals they choose.

- Global Momentum: Steps toward a global flip have already been made by various stakeholders and these experiences can serve as models for the global community. In Europe, a number of transitional offsetting agreements between national institutional consortia and publishers are already in place. The OSI2016 report suggests that organizations of global reach could act as convening authorities in the transition, and the OA2020 Initiative of the Max Planck Digital Library has already gained a significant level of consensus with over 80 signatories representing hundreds of institutions from 26 countries in 5 continents who have committed to making good faith efforts to convert resources currently spent on journal subscriptions into funds that support sustainable OA business models.

- Potential for Cost Savings: While the global flip aims to be, at the very least, cost-neutral for institutions, based on successful results of the SCOAP3 project and empirical data illustrated in the Max Planck Digital Library White Paper, there is evidence that the "global flip" has the potential to lower costs to institutions, with respect to current subscription expenditures, leaving the opportunity for innovation and investment in other, new open access publishing initiatives and services.
- Inclusiveness: In keeping with the premise that a path toward open access that involves all stakeholders will be the most effective, "Proposal 3 " recognizes the unique expertise and key role of all stakeholders in the scholarly communications chain, offering inclusive participation in the transformation.

- Funders will have greater and immediate impact for their investment as research outputs would be immediately open for the benefit of society at large and not subject to embargoes.

- Researchers retain their right to publisher where they choose and control over their copyrighted content.

- Libraries will retain their area of responsibility further developing their strategic and organizational capabilities, and opening up their acquisition budgets to new forms of information and communication services.

- Publishers retain their role in providing publishing services.

- Pressure of Piracy: The rise in alternative, and even illegal access options for researchers places growing pressure on the subscription model, which, in turn, may provide an incentive for publishers to collaborate with other stakeholders toward a scenario that safeguards the integrity of their journals while removing barriers to access.

Conversely, a number of potential challenges to adopting the "Global Flip" strategy were identified:

- Publishers and their journals are global, and approaches to open access must be adopted on a global scale in order to be effective. To be achieved, the global flip requires 
consensus across borders, particularly in regions with a high level of research outputs.

- Some regions-China, for instance-are particularly difficult in the context of a global flip. There are fears that journals involved in the flip could lose researchers from these regions.

- Whereas the proposed pathway would not require new funding or infrastructure, it would require a certain level or reorganization and redirection of revenue streams and workflows.

- Publishers may not be incentivized to embrace such a transition for fear of losing revenues, in particular from markets not directly involved in the production of research output, i.e. corporate subscriptions.

- Changes in economics may also lead to a general sense of uncertainty, for example with regard to job security.

- Certain members of the community propose that self-archiving (Green Open Access) of pre-print versions of research outputs is a sufficient response to the societal demands for open access. This focus on Green can bring with it a reluctance to consider Gold or enter into discussions on how to achieve it.

- There is concern over the potential for an ensuing "pay to publish" model which would be prohibitive to researchers of under-funded institutions as well as the fear of overall cost increases among researchintensive institutions.

- There is a certain amount of distrust between the academic and publisher communities. For example, smaller publishing entities are wary of embarking on a flipped path without having some level of medium-term commitment from institutions.

Some in the academic community, on the other hand, seek new scholarly communications models that omit the need for publishers entirely.

- Differences across subject areas and research types can also pose a challenge to the flip, particularly when considering a business model based on article processing charges (APCs), in light of the relative paucity of grants available in the humanities and social sciences with respect to the STEM fields, and the lack of support for secondary research such as review journals.

- Societies' fears of losing income derived from subscription sales, as well as the loss of a key member benefit contribute to holding back open access generally, and therefore a global flip.

Finally, the question of what impact a global flip would have on the so-called global south raises both challenges and opportunities. The greatest outcome would, of course, be immediate access to the world's scholarly outputs, but subsequent measures would need to be put in place to ensure researchers from these regions and local publishers would have the means to contribute their outputs.

\section{Recommendations}

In order to improve the understanding of the proposed "Global Flip" and its potential impact on goals of the Open Scholarship Initiative, we recommend the following actions:

- Enable further development and dissemination of tools such as the UCPay-It-Forward-Calculation-Tool to increase understanding of the poten- 
tial impact of a global flip on library budgets.

- Commission a third-party study to analyze the financial and scholarly implications of the flip on both publishers and the academic community, starting with an analysis of current research outputs and their costs of publication, dissemination and subscription.

- Propagate results and best practices of key players already involved in the transitional offsetting agreements as part of the global flip strategy. See, for example, the ESAC: Efficiencies and Standards for Article Charges and OpenAPC initiatives, as well as Open Access 2020.

- Identify and support cooperative models that align with the global flip strategy to increase trust and transparency among stakeholders and serve as best practice.

\section{Global Flip \& Other Studies Workgroup}

Eric Archambault, President and CEO, 1science

Colleen Campbell, Director, OA2020 Partner Development, Max Planck Digital Library Lorcan Dempsey, Vice President of Membership \& Research and Chief Strategist, OCLC Roy Kaufman, Managing Director, New Ventures, CCC

Kamran Naim, Lead Researcher, Open Access Cooperative Study, Stanford University; Strategic Development Manager, Annual Reviews

Ralf Schimmer, Head of Scientific Information Provision, Max Planck Digital Library

Wim Van der Stelt, EVP Strategic Relations, SpringerNature

Caroline Sutton, Head of Open Scholarship Development, Taylor \& Francis

Megan Wacha, Scholarly Communications Librarian, City University of New York 